\title{
Remote Sensing of Greenhouse gases by combining Lidar and Optical CORRELATION SPECTROSCOPY
}

\author{
C. Anselmo $^{1 *}$, B. Thomas ${ }^{2}$, A. Miffre ${ }^{1}$, M. Francis ${ }^{1}$, J.P. Cariou ${ }^{3}$, P. Rairoux ${ }^{1}$ \\ ${ }^{1}$ Institut Lumière Matière, CNRS, UMR 5306 Université Lyon 1, 69622 Villeurbanne, France, *Email : \\ christophe.anselmo@univ-lyon1.fr \\ ${ }^{2}$ NOAA CREST Center and Remote Sensing Laboratory, Department of Electrical Engineering, City College, NY, \\ 10031, US \\ ${ }^{3}$ Leosphere France, 14-16 rue Jean Rostand, 91400 Orsay, France
}

\begin{abstract}
In this contribution, we present recent work on the ability to achieve range-resolved greenhouse gases concentration measurements in the Earth's atmosphere $\left(\mathrm{CH}_{4}, \mathrm{H}_{2} \mathrm{O}\right)$ by combining broadband optical correlation spectroscopy (OCS) with lidar. We show that OCSLidar is a robust methodology, allowing trace gases remote sensing with a low dependence on the temperature and pressure-variation absorption cross section. Moreover, we evaluate, as an experimental proof, the water vapor profile in the planetary boundary layer using the $4 v 720 \mathrm{~nm}$ absorption band.
\end{abstract}

\section{INTRODUCTION}

As underlined in the latest IPPC report [1], there is a need for determining the spatial and temporal concentrations of atmospheric constituents. Therefore, Lidar is an efficient monitoring device to probe trace species [2] and also particulate matter [3] coupled with complex atmospheric processes [4]. We have recently developed the OCS-Lidar, a novel instrument that is based on coupling a Lidar with Optical Correlation Spectroscopy (OCS). Its principle and the theoretical approach have then been established [5] leading to a comprehensive study on OCS-Lidar errors budget in the NIR [6]. Moreover OCS-Lidar based on a femtosecond broadband laser source has been successfully applied to retrieve water vapor in the lower atmosphere using the 4v $720 \mathrm{~nm}$ absorption band [5].

This abstract is organized as follows. The OCS-Lidar methodology and the formalism aimed at retrieved the TG concentration are presented in Section 2. Numerical simulations of $\left[\mathrm{CH}_{4}\right]$ remote sensing for extreme pressure and temperature conditions are presented in Section 3. Finally, a field measurement of OCS-Lidar dedicated to water vapor in the planetary boundary layer is presented. The abstract ends with a conclusion.

\section{METHODOLOGY}

OCS-Lidar is an active optical remote sensing method able to monitor atmospheric trace using broadband laser pulse. Its principle is displayed in figure 1.

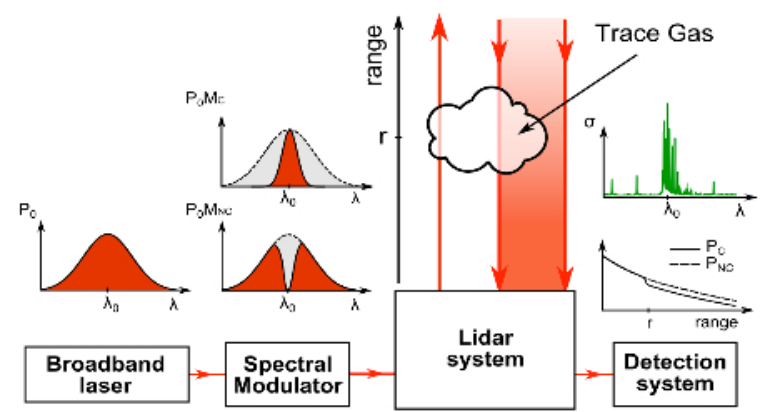

Fig. 1: Scheme of the OCS-Lidar principle.

The broadband laser pulse power density $P_{0}(\lambda)$ is spectrally shaped by alternatively applying two different amplitude modulations $M_{C}$ and $M_{N C}$. A spectral modulator is used to custom one emission laser pulse to be spectrally correlated (C) with the trace gas absorption spectrum while a second one, which is a non-correlated (NC) laser pulse, serves as OCS-Lidar reference signal.

The target gas (TG) concentration profile is retrieved from the signals without the requirement of a permanent calibration gas cell. However a calibration cell could serve to reveal experimental biases. The backscattered OCS-Lidar signal $P_{i}(\mathrm{r})$ can be written as follows:

$$
P_{i}=\frac{K(r)}{r^{2}} \int_{\Delta \lambda} P_{0}(\lambda) \cdot M_{i}(\lambda) \cdot \beta(r, \lambda) \cdot T^{2}(r, \lambda) \cdot \eta(\lambda) d \lambda
$$

where $M_{i}$ is the modulator transmission which ensures the correlation $(\mathrm{i}=\mathrm{C})$ or not $(\mathrm{i}=\mathrm{NC}) . \Delta \lambda$ is the spectral bandwidth of the optical set-up. $\beta(r, \lambda)$ is the atmosphere backscattering coefficient, while $K(r)$ depends on the receiving optics geometry and $\eta(\lambda)$ is the optical set-up efficiency. Finally, $T(\mathrm{r}, \lambda)$ is the transmission of the atmosphere which includes the trace gas (TG) concentration profile. From the ratio of OCSLidar signals $P_{C}(\mathrm{r})$ and $P_{N C}(\mathrm{r})$, we retrieved an analytical expression of $[\mathrm{TG}](\mathrm{r})$ by approximating the TG transmission present in $\mathrm{T}(\mathrm{r}, \lambda)$ with a Taylor expansion series of the Lambert Beer equation. The mathematical description can be found elsewhere [5]. 


\section{EXPERIMENT}

\subsection{Methane}

A numerical model has been developed to generate NIR OCS-Lidar signals and to evaluate the errors budget associated to $\left[\mathrm{CH}_{4}\right]$ remote sensing. It includes detector noise and variation of the Methane absorption spectral lines with the atmosphere thermodynamic changes. Fig. 2 shows, in the form of a color plot, the relative error on the retrieved $\left[\mathrm{CH}_{4}\right]$ by considering the temperature and pressure variations in the troposphere. There, for the input of the model, we consider a 20 ppm Methane peak concentration located at a 1000 meters distance with a 100 meters width range. This numerical simulation [6] shows that the error on $\left[\mathrm{CH}_{4}\right]$ does not overcome $14 \%$ when the temperature and pressure respectively vary by $200 \mathrm{~K}$ and 900 mbar.

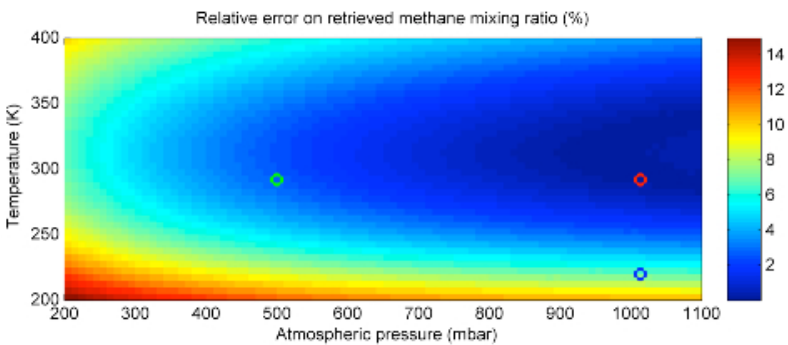

Fig. 2: Relative error in \% on retrieved Methane mixing ratio assuming initial atmosphere temperature and pressure of $292 \mathrm{~K}$ and 1013 mbar respectively.

\subsection{Water Vapor}

The first experimental proof of OCS-Lidar has been performed on the measurement of the water vapor content in the PBL [5].

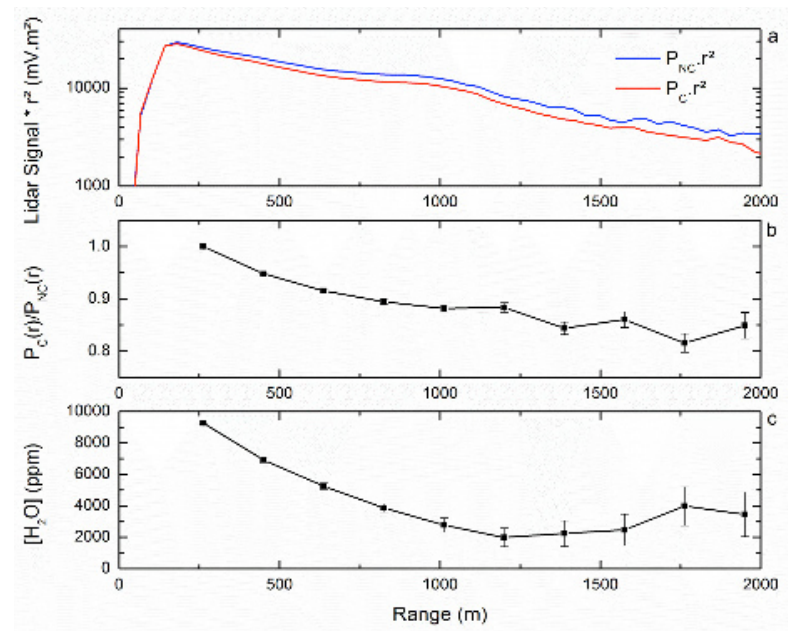

Fig. 3: OCS-lidar water vapor measurement in the PBL. (a) Range-corrected OCS-lidar signals. (b) The ratio $\mathrm{P}_{\mathrm{C}}$ $/ \mathrm{P}_{\mathrm{NC}}$ (c) Retrieved water vapor mixing-ratio profile.
We also demonstrate the ability to realize the experiment using two different configurations of $M_{i}$ (see Eq. 1): on the laser emission or on the reception part. Fig. 3 shows the results of the field experiment when achieving the amplitude modulation $M_{i}(\lambda)$ on $P_{0}(\lambda)$. The water vapor concentration profile in the PBL could be retrieved up to a 2000 meters distance with a $200-\mathrm{m}$ range resolution. The retrieved water vapor mixing-ratio profile starts at $(7,850 \pm 55) \mathrm{ppm}$ at a 260 $\mathrm{m}$ range and reaches $(3,500 \pm 1,000) \mathrm{ppm}$ at the maximum range of 1900 meters. These first experimental results show water vapor mixing-ratio values in agreement with standard atmosphere. Moreover, near ground level $(30 \mathrm{~m})$, in situ hydrometer measurement shows similar values within $15 \%$.

\section{CONCLUSIONS}

We here present the OCS-Lidar based broadband laser source, which is a novel optical methodology to remotely retrieve the concentration of atmospheric greenhouse gases. We demonstrate the ability of this methodology to achieve water vapor filed measurements. Moreover, based on a numerical model, we demonstrate that the OCS-Lidar applied to $\left[\mathrm{CH}_{4}\right]$ detection is almost insensitive to changes in absorption cross-section due to extreme temperature and pressure variations. Future work on $\left[\mathrm{CH}_{4}\right]$ and on other traces gases remote sensing are in progress.

\section{REFERENCES}

[1] IPCC, Climate Change 2013: The Physical Science Basis. Working Group I Contribution to the Fifth Assessment Report of the Intergovernmental Panel on Climate Change (Cambridge University Press, (2013).

[2] C. Weitkamp, "Lidar. Range-Resolved Optical Remote Sensing of the Atmosphere". Springer Ed., (2005).

[3] G. David et al., "Retrieving simulated volcanic, desert dust, and sea-salt particle properties from two / three-component particle mixtures using UV-VIS polarization Lidar and Tmatrix," Atmos. Chem. Phys. 13 (14), 6757-6776 (2013).

[4] David, et al., "UV polarization lidar for remote sensing new particles formation in the atmosphere", Opt. Exp., 22, A1009-A1022, (2014).

[5] B. Thomas et al., "Remote sensing of atmospheric gases with optical correlation spectroscopy and Lidar: first experimental results on water vapor profile measurements," Appl. Phys. B 113(2), 265-275 (2013).

[6] B. Thomas et al., "Remote sensing of methane with broadband laser and optical correlation spectroscopy on the Q-branch of the $2 \mathrm{v} 3$ band," J. of Mol. Spec., 291, 3-8, (2013). 